\title{
Myocardial infarction and quality of care
}

\author{
Jersey Chen MD MPH, Sharon-Lise T. Normand PhD
}

$\infty$ See related research, pages 895 and 901, and related review, page 909

A critical feature of high-quality medicine is that patients receive, or at least to be considered for, all treatments where the benefits outweigh the risks and costs. This is especially true for those with acute myocardial infarction, where a wealth of evidence has led to clinical guidelines with specific indications for therapies. Yet studies report that hospital characteristics, such as teaching status, ${ }^{1}$ or physician characteristics, such as specialty, ${ }^{2,3}$ affect how likely a patient with acute myocardial infarction is to receive evidence-based drug therapies. In this issue of CMAJ, 3 articles remind us of this fact and provide insights to reduce the gap between optimal and actual care for patients with acute myocardial infarction. ${ }^{4-6}$

In the first of 2 articles by Austin and colleagues, ${ }^{4}$ the authors used administrative data to examine the use of $\beta$ blockers, angiotensin-modifying drugs and statins within 90 days after discharge among elderly patients with acute myocardial infarction. Austin and colleagues report that, over a 14-year period, patients were more likely to use these medications if they had received care from a cardiologist, a more recently trained physician or a physician with a high volume of patients with myocardial infarction. The adoption of medications was fastest among cardiologists compared with other physicians, and teaching hospitals had higher use of $\beta$-blockers and more rapid adoption of statins compared with nonteaching hospitals. However, there were only small differences in the rate of adoption of these drug therapies between hospital and physician categories, at most only 3\%-4\% difference. In their companion article, ${ }^{5}$ Austin and colleagues performed a cross-sectional analysis, which reinforced these findings.

What do these 2 articles tell us about the quality of care for patients admitted to hospital for acute myocardial infarction? The percentage of patients who used $\beta$-blockers, angiotensin-modifying drugs and statins after discharge was about $80 \%$ in this study. ${ }^{4}$ In comparison, the levels of use of these medications in the United States are somewhat higher. ${ }^{7}$ However, Austin and colleagues were unable to identify patients for whom drug therapy may have been inappropriate. Because the proportion of such patients varies across physicians $^{3}$ and hospitals, ${ }^{8}$ it is difficult to determine the true difference in performance. The rates of use of drug therapies reported by Austin and colleagues may also be low because their study measured the rate of prescription filling, not prescribing. Prescribing was used in the US study. ${ }^{7}$

\section{Key points}

- The use of evidence-based drug therapies after discharge for treatment of myocardial infarction varies across physicians and hospitals.

- The use of administrative data makes it difficult to determine whether the differences are clinically meaningful.

- Although hospital and physician characteristics explain some of the variation in quality of care, the usefulness of this observation is limited because not all providers with these characteristics perform uniformly well or poorly.

- The 2008 update of the Canadian quality-of-care indicators for acute myocardial infarction will be useful in improving care and outcomes.

What should be done to improve quality of care for patients with acute myocardial infarction? Targeting subgroups of providers is problematic. In the US, there is considerable heterogeneity in the use of evidence-based drug therapies even among high-performing hospitals and physicians. Although studies of cardiologists ${ }^{9}$ and top-ranked hospitals ${ }^{8}$ have reported higher use of acetylsalicylic acid and $\beta$-blockers after acute myocardial infarction, there were physicians who underprescribed these medications in each group. Membership in a hospital or physician group that, on average, performs better than its peers does not ensure that all patients are considered for therapy. In fact, many hospitals and physicians, despite having characteristics that are historically correlated with lower quality of care, provide outstanding treatment.

Patients care most about changes in outcomes such as morbidity and mortality. The studies by Austin and colleagues imply that hospitals and physicians who perform well on measures of care for acute myocardial infarction will also achieve better outcomes. But is this true? Studies have shown that hospitals and physicians with greater use of evidencebased drug therapies for acute myocardial infarction have a limited relation with lower mortality.,10,11 The lack of a strong relation between hospital and physician prescribing of evidence-based drug therapies for myocardial infarction and outcomes suggests that there are other important factors, such as quality of nursing care, nosocomial complications, patient

Jersey Chen is with the Section of Cardiovascular Medicine, Yale University School of Medicine, New Haven, Conn. Sharon-Lise Normand is with the Department of Health Care Policy, Harvard Medical School, and the Department of Biostatistics, Harvard School of Public Health, Boston, USA 
education and follow-up after discharge. Thus, directly measuring outcomes is crucial to make comparisons about the quality of care across providers.

The studies by Austin and colleagues reveal some of the limitations of using hospital and physician characteristics as a tool for improving quality of care for acute myocardial infarction. A comprehensive assessment of quality of care would evaluate patient outcomes, report measures that are hospitalor physician-specific and use clinical data to evaluate the use of evidence-based drug therapies. These concepts are embodied in a third article on this topic in this issue of CMAJ. $\mathrm{Tu}$ and colleagues ${ }^{6}$ summarize the recommendations made by a panel convened to update the 2003 Canadian acute myocardial infarction quality measures. ${ }^{12}$ These quality measures include measures of outcomes, such as mortality and repeat hospital admissions, as well as process indicators based on clinical data. Novel indicators added in the 2008 recommendations include system-wide measures of care, such as the use of prehospital electrocardiograms and the time from call for emergency help to receipt of therapy for myocardial infarction with ST-elevation. These new measures reinforce the philosophy that the quality of health care is a community property that involves many individuals and institutions, not just physicians and hospitals. This is a unique approach that should encourage collaboration across the chain of care for acute myocardial infarction.

The trio of articles in this issue spans a period of transition for the assessment of the quality of care for acute myocardial infarction. We are moving from health policy research that examines the average performance of particular groups of hospitals or physicians to research that investigates providerspecific performance of processes and outcomes. The 2008 update of quality of care indicators for patients with acute myocardial infarction by $\mathrm{Tu}$ and colleagues ${ }^{6}$ is a road map to the future of quality assessment in Canada. This comprehensive model now includes system-wide assessment of quality of care. If successful, this approach offers a viable template for improvements in the quality of care for Canadians admitted to hospital with other conditions.

Competing interests: None declared.

Contributors: Both of the authors contributed to the content of the article, revised it critically and approved the final version submitted for publication.

Acknowledgements: The authors thank Saif Rathore MPH and Harlan Krumholz MD for providing valuable comments on earlier drafts of this editorial.

\section{REFERENCES}

1. Allison JJ, Kiefe CI, Weissman NW, et al. Relationship of hospital teaching status with quality of care and mortality for Medicare patients with acute MI. JAMA 2000;284:1256-62.

2. Frances CD, Shlipak MG, Noguchi H, et al. Does physician specialty affect the survival of elderly patients with myocardial infarction? Health Serv Res 2000;35: 1093-116.

3. Chen J, Radford MJ, Wang Y, et al. Care and outcomes of elderly patients with acute myocardial infarction by physician specialty: the effects of comorbidity and functional limitations. Am J Med 2000;108:460-9.

4. Austin PC, Tu JV, Ko DT, et al. Use of evidence-based therapies after discharge among elderly patients with acute myocardial infarction. CMAJ 2008;179:895-900.

5. Austin PC, Tu JV, Ko DT, et al. Factors associated with the use of evidence-based therapies after discharge among elderly patients with myocardial infarction. CMAJ 2008;179:901-8.

6. Tu JV, Khalid L, Donovan LR, et al. Indicators of quality of care for patients with acute myocardial infarction. CMAJ 2008;179:909-15.

7. Williams SC, Schmaltz SP, Morton DJ, et al. Quality of care in US hospitals as reflected by standardized measures, 2002-2004. N Engl J Med 2005;353:255-64.

8. Chen J, Radford MJ, Wang Y, et al. "America's Best Hospitals" perform better for acute myocardial infarction? N Engl J Med 1999;340:286-92.

9. Frances CD, Go AS, Dauterman KW, et al. Outcome following acute myocardia infarction: Are differences among physician specialties the result of quality of care or case mix? Arch Intern Med 1999;159:1429-36.

10. Werner RM, Bradlow ET. Relationship between Medicare's hospital compare performance measures and mortality rates. JAMA 2006;296:2694-702.

11. Bradley EH, Herrin J, Elbel B, et al. Hospital quality for acute myocardial infarction: correlation among process measures and relationship with short-term mortality. JAMA 2006;296:72-8.

12. Tran CT, Lee DS, Flintoft VF, et al. CCORT/CCS quality indicators for acute myocardial infarction care. Can J Cardiol 2003;19:38-45.

Correspondence to: Dr. Sharon-Lise Normand, Department of Health Care Policy, Harvard Medical School, 180 Longwood Ave., Boston MA 02115, USA; fax 617 432-2563;

Sharon@hcp.med.harvard.edu 